\title{
Understanding the essence and lived experience of self-care management among African-American men living with type 2 diabetes
}

\author{
Ledric D. Sherman, E. Lisako Jones McKyer, John N. Singer, Alvin Larke Jr, Jeffrey J. Guidry \\ Department of Health and Kinesiology, Texas AandM University, College Station, TX 77843-4243, USA
}

\section{A B S T R A C T}

Purpose: To explore, understand and describe the lived experience of African-American men (AA men) living with type 2 diabetes, with emphasis on capturing perceptions of challenges, facilitators and perceived barriers associated with self-care management. Materials and Methods: Participants $(n=19)$ were AA men ages 35-69 years, who were diagnosed with type 2 diabetes. Participants were recruited via community outreach efforts, including barbershops and churches located in predominantlyAfrican-American communities in southeast US. Upon consent, individual interviews were conducted, audio-recorded and subsequently transcribed. Transcripts were analyzed using a phenomenological approach, and focused on identifying common themes among the descriptions of AA men's experiences specific to type 2 diabetes. Results: AA men perceived their experience of managing type 2 diabetes as tedious, complicated, demanding, and frustrating. Common themes included the perception of family histories and personal behaviors as causes or contributors to the development of diabetes, albeit there was lack of clarity regarding biological versus behavioral familial contributions. Other theses included fears related to long-term complications of type 2 diabetes, and the critical role of social support as a factor assisting with selfcare management. Limb amputation, insulin injections and vision changes were fears related to having type 2 diabetes. Commensurately, important referent others (e.g., family and close friends) provided critical encouragement and support toward managing their diabetes. Conclusions: Future diabetes research and education should give attention to how masculinity may have a powerful influence on diabetes management behavior among African-American men's as well as utilizing preventive health services.

Key words: African-American Men, self-care management, type 2 diabetes

\section{INTRODUCTION}

"Actually, it's [type 2 diabetes diagnosis] been a Godsend because it's [dieting and exercise] something that you need to do anyway... so I don't look at it [diabetes diagnosis] as being that negative."

There is a sizable gap in health disparities and public health literature addressing the self-care management

\begin{tabular}{|l|l|}
\hline \multicolumn{2}{|c|}{ Access this article online } \\
\hline Quick Response Code: & Website: \\
\hline & www.josh.net \\
\hline & \\
\hline
\end{tabular}

experiences of African-American Men living with type 2 diabetes. Type 2 diabetes can be a challenge to manage, as most of the care requires significant levels of selfmanagement. Management of type 2 diabetes focuses on lifestyle interventions, lowering other cardiovascular risk factors, and maintaining blood glucose levels in the normal range.

In the United States, great health inequities exist for racial/ethnic minority groups, compared to white Americans (WA).African-Americans, and in particular African-American Men (AA men) suffer disproportionate burden of many acute and chronic conditions. ${ }^{[1]}$ Specific to type 2 diabetes, while prevalence rates for all men irrespective of race/ethnicity is about $11.8 \%$, prevalence of type 2 diabetes is $17.6 \%$ among AA men ages $45-64$ years, compared to their white counterparts $(12.4 \%){ }^{[2]}$

Corresponding Author: Dr. Ledric Sherman, 4243 TAMU, College Station, TX 77843-4226, USA.

E-mail: ledricsherman@gmail.com 
Self-management studies indicate that AA men are significantly less adherent to medication regimen than nonHispanic WA men. ${ }^{[3]}$ Consequently, AA men have 30\% higher rates of diabetes-related blindness, and twice as many amputations compared to their white counterparts..$^{[4]}$ Clearly, type 2 diabetes can become a staggering ailment for this group if attention is not focused on treatment and maintenance.

\section{What is self-care management?}

Diabetes self-management is defined as the knowledge, and skills needed to perform the self-care, manage crises, and make the lifestyle changes required to successfully manage the disease. ${ }^{[5]}$ Successful management of type 2 diabetes requires daily attention to a complex adaptation of behaviors such as healthy eating, medication adherence, stress management, blood glucose monitoring and testing, management of hypoglycemia, physical activity, and foot care..$^{[5]}$

\section{Rationale forthe focus on african american men} While both genders and all ethnicities are confronted by the continual necessity of type 2 diabetes management, there is insufficient literature centered on AA men and the contexts in which they go about living and managing the disease. In spite of the plethora of information available regarding the adverse outcomes of poor management of type 2 diabetes among AA men, there is still a paucity of information from their perspective regarding extra challenges related to diabetes self-management. There are signs that researchers are beginning to realize the importance of focusing on AA men. ${ }^{[6-18]}$ Yet, the uniqueness of the AA men's experiences as a disenfranchised group in the U.S. requires efforts to understand factors specific to these men and the contexts in which they live and operate. This includes considering factors such as masculinity, mistrust, and how these are related to diabetes knowledge, fatalism, medical decision making, and the psychosocial impacts of self-management among AA men. Understanding a population in regard to self-management practices is likely important to improved self-management support and ultimately chronic illness care. ${ }^{[19]}$

\section{Study purpose}

The primary purpose of this study is to gain an understanding of the lived experiences of AA men living with type 2 diabetes. We sought to clarify this from the perspective of the subject. Two sub-questions were to identify and clarify:1) What barriers or challenges AA men face regarding positive self-care management?, and 2) What management methods do these men routinely incorporate in their lives?.

This article extends the literature by providing information on AA men's individual and very personalized experience of how type 2 diabetes has impacted their lives, their experience in managing type 2 diabetes, and the challenges they confront in self-care management. Findings can provide clarity about which diabetes care measures and outcomes are relevant for AA men - a marginally understood group - and lead to improved interventions to facilitate better disease management.

\section{MATERIALS AND Methods}

\section{Ethics and the protection of human subjects}

The study protocol was reviewed for adherence to ethical research principles, including steps to ensure participant confidentiality and other factors related to the protection of human subjects in research, as required by the U.S. Department of Health and Human Services. The protocol was approved by the federally-mandated Institutional Review Board (IRB) at the major research university in which the authors are based.

\section{Study design}

\section{Selection and description of participants}

Target population was AA men with physician diagnosis of type 2 diabetes, who reside in a southern state of the U.S. The inclusion criteria were: 1) participant was self-identified as African-American; 2) age 18 to 70 years; 3) physician-diagnosed with type 2 . Each participant selected their interview location. Most chose to conduct their interview at their place of residence. Two of the 19 participants chose to have their interview at their place of employment due to convenience factors. At each interview location, the interviewer and participant sat face to face in a private location.

The derived sample included AA men $(N=19)$, ages 35-69, with type 2 diabetes in southeast Texas. The age limit on the higher end was set as part of an attempt to include men who had been medically diagnosed with type 2 diabetes, but to exclude those who were living with and/or managing any other major chronic illness as it could confound the aims of the study. A communityfocused recruitment strategy was used; Establishments with high proportions of African American clienteles (e.g., barbershops, urban churches) were targeted. This strategy was combined with snowball sampling, which is used when researchers are coping with issues of access and sensitivity of the topic. ${ }^{\text {[20] }}$

\section{Phenomenological methodology}

The focus of a phenomenological study lies in the "descriptions of what people experience and how it is that they experience" ${ }^{[21,22]}$ with the goal to identify essence (i.e., commonalities) $)^{[22,23]}$ of the shared experience underling 
variations in this particular experience. This methodis used to study areas in which there is little knowledge. ${ }^{[21-23]}$ It is especially relevant to type 2 diabetes as it allows us to "extrapolate meaning of the illness from the participant's perspective."[24]

Describing the data collector/researcher is the key part of phenomenology methodology ${ }^{[22,23]}$ For this study, the lead researcher also served as the primary data collector, and is an AA male with a research doctorate in health education and expertise in qualitative research methods.

\section{Instruments and data collection}

The lead researcher carried out the following protocol: (a) read the consent form, discussed participants' rights, and obtained informed consent, (b) requested the participant to complete the demographic profile questionnaire, and (c) performed the face-to-face recorded interview. Interviews lasted approximately 35 to 90 minutes. Each participant was given the option to end the session at any time without penalty. Participants'incentives included a \$25 gift card, and a brief report of the findings upon completion of the study.

\section{Instruments}

Brief Demographic Questionnaire was utilized to collect relevant information about each participant, including their age, marital status, education level, household income, and how long they have been living with type 2 diabetes. Accompanying questions pertained to diabetes -specific medical visits and educational session attendance.

Semi-structured Interview Guidedeveloped according to the most recent type 2 diabetes research relating to AfricanAmericans. Criterion validity was established via review and consultation by two experts: 1) a qualitative methodologist with expertise specific to African-American men, and 2) a chronic disease and health inequities researcher. The guide [Table 1] concentrated on eliciting data from six areas: Diabetes management practices, knowledge and beliefs about diabetes, perceived barriers to diabetes management, social support, symptoms, and personal vs. interpersonal feelings regarding diabetes management.

For this paper, we focus on responses to items 2, 3, 5, and 6-9 as these were design around the study's specific sub-questions.

\section{Data analysis}

Narrative analyses has been used to explore life experiences of individuals with diabetes. ${ }^{[24]}$ Fidelity to the phenomenon as it is lived means capturing and understanding it as perceived through the person living the situation. ${ }^{[25]}$
Participants in this study told their own story and in their own terms. Therefore, excerpts from their transcripts were not edited or corrected, and are presented in their unique voice as originally recorded.

Themes and patterns are sought, both during and a priori data collection. The lead investigator transcribed all interviews and performed several rounds of active listening. The analyses included personal memos (PN)-personal ideas or reflectionswhich emerged, and theoretical notes (TN) -where transcriptdata reflected relevant ideas or concepts. Data were then coded (i.e., labeled) and aggregated into themes which are defined as "something important about the data in relation to the research question and [something] that represents some level of patterned response."[26] Themes were derived from the brief codes, using memos and structures identified in the previous steps. Finally, statements from participants' transcripts that best answered the interview questions were selected. Specifically, the narrative emerged from illustrative data extracts arranged according to the section headings of the semi-structured interview guide, and then organized according to the primary research question and two sub questions. Emergent themes which did not fall under the three primary questions were considered new findings.

\section{RESULTS}

\section{About the participants}

The final sample consisted of 19 AA men, of which most (68.4\%) were over age 55 years. About $66 \%$ had lived with diabetes for 10 years or less. Table 2 fully details relevant socio-demographic characteristics of the final sample.

\section{Participants etiology beliefs: Developing diabetes}

Two categories of beliefs related to diabetes etiology were derived from the interviews.Participants believed that family history and their own personal health-

\section{Table 1: Sample interview guide questions}

1) When your doctor first told you that you had developed type 2 diabetes, how did it make you feel?

2) What do you think caused you to have developed diabetes?

3) Do you have any fears about having type 2 diabetes?

4) What changes did you notice about your body that may have led to your diabetes diagnosis?

5) Have you experienced any other problems because of your diabetes?

6) What kind of treatment(s) do you prefer to help you manage your diabetes?

7) Describe how you control your diabetes.

8) Is there someone in your life who helps you control your diabetes?

9) What do you feel are the most difficult diabetes management behaviors for you to do on consistent basis?

10) Do you feel that you have more stress in your life since you found out you have type 2 diabetes? 


\begin{tabular}{|c|c|}
\hline Table 2: Summary of participa & $\mathrm{N}=19$ ) \\
\hline $\begin{array}{l}\text { Age Range } \\
35-44 \\
45-54 \\
55-64 \\
65-\text { older }\end{array}$ & $\begin{array}{l}\% \\
2(11) \\
4(21) \\
6(32) \\
7(37)\end{array}$ \\
\hline $\begin{array}{l}\text { Annual Income } \\
<25,000 \\
25,000-34,999 \\
35,000-49,999 \\
50,000-74,999 \\
75,000-99,999 \\
100,000-149,999 \\
>150,000\end{array}$ & $\begin{array}{l}2(11) \\
2(11) \\
1(5) \\
4(21) \\
2(11) \\
4(21) \\
4(21)\end{array}$ \\
\hline $\begin{array}{l}\text { Marital status } \\
\text { Single } \\
\text { Married } \\
\text { Divorced } \\
\text { Widowed }\end{array}$ & $\begin{array}{c}2(11) \\
14(74) \\
2(11) \\
1(5)\end{array}$ \\
\hline $\begin{array}{l}\text { Education Level } \\
<\text { high school } \\
\text { some college but no degree } \\
\text { associate's degree } \\
\text { bachelor's degree } \\
\text { graduate or professional degree }\end{array}$ & $\begin{array}{l}1(5) \\
7(37) \\
3(16) \\
5(26) \\
3(16)\end{array}$ \\
\hline $\begin{array}{l}\text { Living with T2DM } \\
\text { 1-5 yrs } \\
6-10 \text { yrs } \\
11-15 \text { yrs } \\
16-20 \text { yrs } \\
>20 \text { years } \\
\text { Don't know }\end{array}$ & $\begin{array}{l}7(37) \\
5(26) \\
2(11) \\
3(16) \\
1(5) \\
1(5)\end{array}$ \\
\hline $\begin{array}{l}\text { Attended educational classes } \\
\text { Yes } \\
\text { No }\end{array}$ & $\begin{array}{c}9(47) \\
10(53)\end{array}$ \\
\hline $\begin{array}{l}\text { Doctor visits per year } \\
\text { 1-2 times per year } \\
\text { 3-4 times per year }\end{array}$ & $\begin{array}{c}8(42) \\
11(58)\end{array}$ \\
\hline
\end{tabular}

related behaviors were at the root of their disease development.

\section{Family history}

Participants were cognizant of an association between family history of type 2 diabetes, and the development of their own diagnosis. Theydiscussed family history (maternal vs. paternal lineages)as contributors. However, not all participants understood the differences between biological, genetic us. social/behavioral influences on their diabetes, as reflected in the following excerpts:

Well... it's a product of my environment because everyone that's in my family either has high blood pressure, diabetes, asthma, so... I guess it's just in my genes."

"It's hereditary in my family. I found out that my sister has it... and she was a type 1 diabetic... I found... that another sister had it... I found out by talking to them that it's hereditary."
"Umm... like I said hereditary, it's something that you know, if it's on your mothers' side or fathers' side, it's not like something that you just go out and catch. It's just in your genes or whatever from your mother or father and for me, it just so happened to be from my fathers' side of the family. So that's why."

\section{Personal Health Risk Behaviors}

Participants' perceived factors under their volitional control may have contributed to the onset of type- 2 diabetes. Participants mentioned diet (e.g., lack of vegetables) and health behaviors (e.g., sedentary behaviors, eating behaviors, tobacco and alcohol use) as examples.The following excerpts highlight this perspective:

"For me, it was diet and the fact I wasn't getting enough exercise. I think both of those for sure."

"It probablystems from a long history of improper feeding. You had to have what you had and a lot of sugar was involved at that time. So I think that was probably one of the major causes. We ate a lot of potatoes and starchy stuff... stuff like that because we didn't know any better."

"Bad habits...eating, consuming liquids (laughing), the wrong kind of liquids... can I say alcohol? (laughing) Yeah, alcohol."

"Family possibly had a lot to do with it, but I did also. In my younger years ... I didn't drink a lot of water, I was drinking Cokes. .. . That I think was the contributing factor."

\section{Fears associated with type 2 diabetes}

There were two types of fears related to their disease as reported by most of the AA men, regardless of well or poorly they were managing their disease. These fears were related to the long-term effects of the diabetes, rather than acute episodes of symptoms related to poor management.

\section{Adverse health consequences}

The loss of one or more body function, or permanent change in their physiology, including amputations, kidney and vision failure, as well as sexual dysfunction, were fears reported by these men.

"I say fear of eyesight because I've always been near-sighted since a child so I've always had contacts, glasses, something like that. And then with diabetes, that can affect your eyesight as well."

"Dialysis, kidney failure, which would require you to be on dialysis, loss of limbs, eyesight, and other serious causes of diabetes."

"Yes. Loss of vision, Loss of limbs, Inability to perform sexually." 


\section{Management-related fears}

AA men whose condition did not require insulin shots reported their fear of the injections, including how it would serve as an indicator of disease progression, and would further complicate the self-care management regimen.

"I hope that I don't have to end up taking insulin shots. My biggest fear is taking insulin shots. That's the biggest one..."

"I can do it ... as long as needles aren't involved... I can't stand needles."

\section{Lack of Fear}

A few participants stated they did not have any fears related to their condition, and credited improved knowledge as the cause. Formal diabetes management education, and witnessing others who were effectively managing their diabetes was effective for these men in alleviating their concerns related to the disease.

"[have any fears?] Not anymore. Now that I've studied it, done research on my own. I know the do's and don'ts and I know that it can be handled. I don't have any fear at all."

"Like I said before I was sad because of some things that I heard from others about it. But once I started reading about and talking to different people that have it, it's a better chance of living longer when you're eating right and maintaining the way you eat. I feel more energy and more happier just by knowing more about it."

\section{Diabetes-attributed problems}

Participants noticed changes in their bodies that were not in existence prior to being diagnosed with type 2 diabetes. Commonly held beliefs were that these changes were due to imbalances in their bodies, including unstable and elevated blood sugar levels.Vision difficulties, as well as frequent urination were the most common. AA men were cognizant of improvements and even elimination of these symptoms, and attribute this to good care from their physicians.

"I have these spots... little black spots that come over my vision... it seems that I have more little spots that come on chest, shoulders, and arms and when I asked my doctor about that, she said that was a result of diabetes."

"Having to get up and got the restroom more often, I'm attributing that to diabetes. However, since the doctor put me on this medication, that's no longer an issue."

"Yes. The tingling in the fingers and the tingling in the feet and things like that and I ended up having to take medication for that."
". . problems with my eyesight until I started going to the eye doctor regularly. So I go to my eye doctor every three or four months too.. . so I take eye medication now and that has helped me improve me tremendously."

\section{Treatment preferences}

Participants' shared two types of treatment preferences: Medication, and behavioral (e.g., dietary changes, and increase in exercise).

\section{Medication}

In some participants' experiences, medication was preferable over behavioral changes (e.g, diet, exercise) as a type 2 diabetes management strategy.

"After going through this for a long as I have, the pills would probably be the easiest because there's no injections, no pain."

"My doctor has put me on medicine... a little bity pill, one pill a day and that's all I take... that's all I have to take for diabetes. And it seems to be doing pretty good."

\section{Behavioral strategies}

AA men shared in common their struggle to initiate and maintain behavioral changes necessary to effectively manage their type 2 diabetes. This theme persistedacross educational levels, and in spite of behavioral approaches being their preferred mode of management.

"I try to minimize eating sweets and having as proper of a diet as possible. I need to incorporate so more exercises in my life which is something that I've been thinking about. The hardest about that is trying to be motivated."

"Other than exercise, I have gone to one or two diabetic classes to learn more about a balanced diet for diabetics and I have lost weight in the past. My biggest change is cutting sweets out. I love sweets, but I have cut back... very seldom do I have them."

\section{Control and monitoring of diabetes}

Various strategies were shared by the AA men in their attempts to monitor and control their type 2 diabetes. Common approaches were attempts to increase water intake, dietary changes, and steps to improve routine control, close attention and monitoring of their blood sugar levels.

"I eat small amounts. I eat breakfast before going to work so I don't get tired. I make sure I have lunch. I also take crackers with me to eat in between because you never know if your blood sugar will get to low or get to high... you want to know how to manage that. And every morning, I always check my glucose. Even if I don't feel right, I check it." 
"First thing I do in the morning is I have to take my pill. Then for breakfast, it's only two things that I eat, oatmeal or Cheerios, Honey Nut Cheerios. And then I have to eat fruit every day and a little salad. But my biggest adjustment was sweets."

\section{Assistance with controlling diabetes}

Strong support system was a common experience among the participants, in terms of assisting them in managing their diabetes. Support network membership was primarily comprised of relatives. The types of support offered varied from informational and instrumental (e.g,joint exercise, appointment reminders, monitoring sugar intake, purchasing educational materials) and emotional (e.g., encouragement).

"My wife. She's ordered a whole series of cookbooks and other books that talks about diabetes and ... on TV . . because she's trying to keep me around here longer. . she's been encouraging me."

"My wife goes with me to every visit. . . That is encouraging in itself. And I want her there and she listens to what the doctors say."

"My brothers of course... although we live in different geographical locations, we talk a lot on the phone and we encourage each other and check with each other. And since I have one brother that is diabetic, we check and talk to each other a lot.

"Yes, my wife definitely... she buys groceries. So she shops and like I said before, she has the patience to check for carbohydrates in the foods and different things like that."

\section{Difficult management-related behaviors}

There were three main behaviors found to be difficult to consistently perform among the participants: Exercise, dietary changes, and taking medication. They attributed the challenges with implementing and maintaining behavioral management strategies to the time conflicts, as well as their advancing age.

\section{Exercise}

"My biggest thing is the exercise part... the doctor, they say...you need to exercise more... lose weight. If I can start doing that more, I can probably reduce my medicine..."

\section{Dietary changes}

"Sticking to the diet... eating healthy all of the time. Eating healthy doesn't have any taste... it doesn't. And for so long I've eaten the way I've eaten so it's just curving that. And then I have spells where I'll go eat sweets... I'll eat a lot of sweets."

"The drinking (alcohol). I'm not saying I'm alcoholic but I love to drink. Every now and then it's like I'll go on a binge and start drinking a beer or something... I can't just have one. Where as
I know alcohol is not a good part of the diet, but I guess we all have some demons that we have to deal with (laughing). If I drink them margaritas, then I got a problem."

\section{Medication adherence}

"Me, I hate medications. I truly, truly hate medications... never been the type of person to take medication unless I was ill, which I know that I am having diabetes. ... I've just never been a pill popping guy.I only take it if it's absolutely necessary."

\section{Age and time}

"As you get older, your body is not like it was 20 years ago and so your body can't respond to stuff at times..."

"Taking the time and putting it in a routine is what makes it hard to do consistently."

\section{DISCUSSION}

\section{Summary of key findings}

Historical and current marginalization of AA men in the US, reflected by tremendous health inequities, renders important the need to gain an understanding of their experiences, particularly in relation to their diabetes selfmanagement activities. Thus, the purpose of the study was to identify via analyses of narrative interviews, the experiences of AA men with type 2 diabetes, with the aim of elucidating their voices - i.e., their perspectivescontextualized in their respective lives.

We made special effort to identify barriers and challenges associated with self-care management, as well as an exploration of the various strategies used by these men. We found that family history, heredity, and lifestyle choices were stated by over half of the participants, to be the cause of their type 2 diabetes.Family history is crudely defined as the presence of disease in any family member irrespective of the degree or type of relatedness or number of affected relatives. ${ }^{[27]}$ Family histories reflect both inherited genetic responsibilities and shared environments, which include cultural factors such as preferences, values, and perceptions and behavioral factors such as diet and physical activity. ${ }^{[28]}$

It was evident that participants did not always distinguish between the social and biological types of family history contribution toward their disease status. While the lack of knowledge regarding disease etiology might appear to be a concern, we argue that the lack of etiological understanding need not be a barrier to improved adherence to the prescribed regimen. We conclude this based upon the multi-functional roles family members play in helping AA men manage their diabetes. 
Family and close friends can generate a positive environment for AA men with diabetes, via the provision of informational and instrumental support, as well as emotional support.Participants in this study struggled with managing their diabetes, yet all relied on the encouragement of their family. These findings are constant with the results of other studies ${ }^{[29,30]}$ in which family and friend support was critical to assist in the management of diseases.

Family members can also hamper successful disease management by modifying the context in which this takes place. For example, AA men's efforts to improve their diets could be sabotaged if family members were not sensitive to dietary needs and continued to practice eating behaviors which were contraindicated for those with diabetes. Therefore, regardless of whether the "family history" was biological or behavioral, the real potential for familial influence - whether positive or negative exists. As a consequence, less attention needs to be paid to helping men understand the difference between genetic versus behavioral family contributions to the disease, and more attention paid to how familial social support can and should be a target forAA men reported fears related to their disease, regardless of how well they were managing their health. There is a significant body of literature on the diabetes management and "fear." However, a published review of the literature reveals the foci of these studies are on fears specific to the adverse effects of hypoglycemia ${ }^{[31]}$ which is typically associated with type 1 diabetes. Symptoms of hypoglycemia include (but are not limited to) dizziness, shakiness, seizures, hunger and nausea. ${ }^{[31]}$ Other than concerns about vision impairments, none of our participants included hypoglycemia symptoms as concerns. Indeed, their concerns about visions were related to the long-term complications of diabetes related to compromised circulation, rather than vision issues brought about by an acute episode related to poor diabetes management. Furthermore, while hypoglycemia is usually of concern for people with type 1 diabetes, any person on an insulin regimen is at risk. ${ }^{[31]}$ Our findings point to the need to better understand and address fears related to type 2 diabetes which are unique to AA men.

\section{Study strengths and limitations}

The qualitative approach, and in particular, the phenomenological approach is a strength of this study. Stuckey ${ }^{[24]}$ noted the utility of this methodological and conceptual approach toward studies such as this one, where very little is known in general about AA men's life experiences, and even less is known about those who are trying to manage a chronic disease such as type 2 diabetes.
The type of sampling and method/design used does not lend itself to generalizability.Some may argue about the utility of our findings. We argue that our results provide an important starting point from which more specific questions, using appropriate strategies, should be pursued. Indeed, a qualitative approach is and should always be the starting point for any research question in which virtually no data are available. ${ }^{[20-22]}$

One limitation may be the absence of explicit queries or references to issues of race, ethnicity and gender relative to AA men and their experiences. AA men in the US tend to be marginalized, as reflected in a variety of adverse socioeconomic and health indicators. These inequities are believed to be due, in part, to institutionalized racial bias against Americans of African descent in general, ${ }^{[32]}$ with perhaps an exponential effect on men. A previous study seeking to determine if culture and genders-specific theories and constructs were used in type-2 diabetes selfmanagement research were revealed that zero studies met the criteria. ${ }^{[33]}$ This means that while "culture" as a generic construct might be included in health behavior theories and models, it has not been defined, operationalized with AA men in mind, nor integrated in research pertaining to them and their efforts to self-manage type 2 diabetes.

Of additional note and contextual information, African Americans, and AA men's distrust of the medical and health-related community is well established ${ }^{[34-36]}$ due in part to well-known incidences of African Americans being exploited and harmed (e.g., Tuskegee Syphilis Study). Consequently, health and medical researchers struggle today with recruiting and retaining African Americans in research.

We attempted to address potential bias by having the lead researcher - an African American male - serve as the primary liaison and data collector. However we did not explicitly ask about issues related to race, ethnicity or masculinity, or ask the participants to contextualize their diabetes self-management experiences as AA men. This may serve as a limitation as the participants might not have perceived their interviews as a chance to tell their stories specifically from the perspectives as AA men.

Implications

Our study contributes to the extant research literature on self-management of type 2 diabetes by shedding light on the facilitators and barriers of diabetes selfmanagement strategies utilized by AA men. There is so little information available about AA men and virtually none from their perspectives. Our results highlight the need to explore more deeply and specifically, how these 
factors can be used to improve diabetes self-management practices.

\section{Future research}

Future research efforts should focus on three primary areas: Clarify how AA men's experiences related to 1) their positions as racial/ethnic minorities in the US, and 2) their gender identity, definitions and perceptions of masculinity, are related to their self-management behaviors. These can be achieved using a similar methodology as the present study, but with the addition of explicit questions related to their racial minority status and of manhood or masculinity. The aim of these efforts should be to clarify if and how the experiences of being a marginalized member are perceived to have influence on disease self-management efforts. Third, there is a need to further understand "fear" factors, as the ones reported by our participants appear to differ from those in the extant research literature.

\section{ACKNOWLEDGEMENT}

This study was funded, in part, by the TransdisciplinaryCenter for Health Equity Research (TCHER). TCHER is housed in the Department of Health and Kinesiology, College of Education and Human Development, at Texas A\&M University. We gratefully acknowledge the study participants who gave of their time and showed us patience throughout the process.

\section{REFERENCES}

1. LaVeist TA, Isaac L, editors. Race, Ethnicity and Health: A public health reader. $2^{\text {nd }}$ ed. San Francisco, CA: Jossey-Bass; 2013.

2. Centers for Disease Control and Prevention: National Diabetes Surveillance System. Age-Specific Percentage of Civilian, Noninstitutionalized Population with Diagnosed Diabetes, by Age, Race and Sex, United States, 2011. Available from: http:// www.cdc.gov/diabetes/statistics/prev/national/fig2004.htm [Last accessed on 2013 Nov 19].

3. Shenolikar RA, Balkrishnan R, Camacho FT, Whitmire JT, Anderson RT. Race and medication adherence in Medicaid enrollees with type-2 diabetes. J Natl Med Assoc 2006;98:1071-7.

4. Hendricks LE, Hendricks RT. The effect of diabetes selfmanagement education with frequent follow-up on the health outcomes of African American men. Diabetes Educ 2000;26:995-1002.

5. Clement S. Diabetes self-management education. Diabetes Care 1995;18:1204-14.

6. Hammond WP, Matthews D, Mohottige D, Agyemang A, Corbie-Smith G. Masculinity, medical mistrust, and preventive health services delays among community-dwelling AfricanAmerican men. J Gen Intern Med 2010;25:1300-8.

7. Liburd LC, Namageyo-Funa A, Jack Jr L, Gregg E. Views from within and beyond: Illness narratives of African-American men with type 2 diabetes. Diabetes Spectr 2004;17:219-24.

8. Hart Jr A, Smith WR, Tademy RH, McClish DK, McCreary M. Health decision-making preferences among African American men recruited from urban barbershops. J Natl Med Assoc 2009;101:684-9.
9. Chesla CA, Fisher L, Mullan JT, Skaff MM, Gardiner P, Chun $\mathrm{K}$, et al. Family and disease management in African-American patients with type 2 diabetes. Diabetes Care 2004;27:2850-5.

10. Peek ME, Wilson SC, Gorawara-Bhat R, Odoms-Young A, Quinn MT, Chin MH. Barriers and facilitators to shared decision-making among African-American with diabetes. J Gen Intern Med 2009;24:1135-9.

11. Peek ME, Quinn MT, Gorawara-Bhat R, Odoms-Young A, Wilson SC, Chin MH. How is shared decision-making defined among African-Americans with diabetes? Patient Educ Couns 2008;72:450-8.

12. Baptiste-Roberts K, Gary TL, Bone LR, Hill MN, Brancati FL. Perceived body image among African-Americans with type 2 diabetes. Patient Educ Couns 2006;60:194-200.

13. Sanders Thompson VL, Talley M, Caito N, Kreuter M. African American men's perceptions of factors influencing health-information seeking. Am J Mens Health 2009;3:6-15.

14. Jack Jr L, Totson T, Jack NH, Sims M. A gender centered ecological framework targeting Black men living with diabetes: Integrating a "masculinity' perspective in diabetes management and education research. Am J Mens Health 2010;4:7-15.

15. DeWalt DA, Boone RS, Pignone MP. Literacy and its relationship with self- efficacy, trust, and participation in medical decision making. Am J Health Behav 2007;31:S27-35.

16. McCleary-Jones V. Health literacy and its association with diabetes knowledge, self-efficacy and disease self-management among African-Americans with diabetes mellitus. ABNF J 2011;22:25-32.

17. Bhattacharya B. Psychosocial impacts of type 2 diabetes self-management in a rural African-American population. J Immigr Minor Health 2012;14:1071-81.

18. Walker EA, Stevens KA, Persaud S. Promoting diabetes self-management among African-Americans: An educational intervention. J Health Care Poor Underserved 2010;3:169-86.

19. Clark DO, Frakel RM, Morgan DL, Ricketts G, Bair MJ, Nyland KA, et al. The meaning and significance of self-management among socioeconomically vulnerable older adults. J Gerontol B Psychol Sci Soc Sci 2008;63:S312-9.

20. Schutt RK, editor. Investigating the social world: The process and practice of research. $7^{\text {th }}$ ed. Thousand Oaks, CA: Sage Publications; 2012.

21. Patton MQ. Qualitative evaluation and research methods. $2^{\text {nd }} \mathrm{ed}$. Newbury Park, CA: Sage Publications; 1990.

22. Creswell JW. Qualitative inquiry and research design: Choosing among five approaches. $2^{\text {nd }}$ ed. Thousand Oaks, CA: Sage Publications; 2007.

23. Donalek JG. Demystifying nursing research: Phenomology as a qualitative research method. Urol Nurs 2004;11:218-25.

24. Stuckey HL. Three types of interviews: Qualitative research methods in social health. J Soc Health Diabetes 2013;1:56-9.

25. Moustakas C. Heuristic research: Design, methodology, and applications. Newbury Park, CA: Sage Publications; 1990.

26. Braun V, Clarke V. Using thematic analysis in psychology. Qual Res Psychol 2006;3:77-101.

27. Anderson BJ. Involving family members in diabetes treatment. In: Anderson BJ, Rubin RR, editors. Practical Psychology for Diabetes Clinicians. Alexandria, VA: American Diabetes Association; 1996. p. 43-50.

28. Keku TO, Millikan RC, Martin C, Rahkra-Burris TK, Sandler RS. Family history of colon cancer: What does it mean and how is it useful? Am J Prev Med 2003;24:170-6.

29. Miller C, Davis M. The influential role of social support in diabetes management. Top Clin Nutr 2005;20:157-65.

30. Cohen S. Psychology models of the role of support in the etiology of physical disease. Health Psychol 1988;7:269-97. 
31. Wild D, von Maltzahn R, Brohan E, Christensen T, Clauson P, Gonder-Frederick L. A critical review of the literature on fear of hypoglycemia in diabetes: Implications for diabetes management and patient education. Patient Educ Couns 2007;68:10-5.

32. Williams DR, Mohammed SA. Racism and Health 1: Pathways and scientific evidence. American Behavioral Scientist 2013;DOI: $10.1177 / 0002764213487340$.

33. Sherman LD. "Blood sugar and brothers' voices: An exploratory study of the self-care management experiences of black men living with type 2 diabetes. (unpublished doctoral dissertation). Texas A and M University, College Station, TX, 2013.

34. Gamble VN. Under the shadow of Tuskegee: African Americans and health care. Am J Public Health 1997;87:1773-8.

35. Jacobs EA, Rolle I, Ferrans CE, Whitaker EE, Warnecke RB. Understanding African Americans' views of the trustworthiness of physicians. J Gen Intern Med 2006;21:642-7.

36. Kennedy BR, Mathis CC, Woods AK. African Americans and their distrust of the health care system: Healthcare for diverse populations. J Cult Divers 2007;14:56-60.

How to cite this article: Sherman LD, McKyer EL, Singer JN, Larke A, Guidry
JJ. Understanding the essence and lived experience of self-care management
among African-American men living with type 2 diabetes. J Soc Health Diabetes
2014;2:96-104.
Source of Support: Nil. Conflict of Interest: None declared. 